Abanico Veterinario. Enero-Diciembre 2020; 10(1):1-10. http://dx.doi.org/10.21929/abavet2020.2 Artículo Original. Recibido: 25/02/2019. Aceptado: 16/09/2019. Publicado: 06/01/2020.

\title{
Actividad antibacteriana del extracto hidroalcohólico Croton draco sobre bacterias de importancia sanitaria
}

\author{
Antibacterial activity of the hidroalcoholic extract Croton draco on bacteria of sanitary \\ importance
}

\section{Morales-Ubaldo Ana ${ }^{1}$ (D), Hernández-Alvarado Jerelly ${ }^{2}$ (D) , Valladares-Carranza Benjamín $^{3}$ (iD), Velázquez-Ordoñez Valente ${ }^{3}$ (iD), Delgadillo-Ruiz Lucía ${ }^{4}$, Rosenfeld- Miranda Carla ${ }^{5}$ D , Rivero-Pérez Nallely ${ }^{1^{*}} \stackrel{(D)}{ }$, Zaragoza-Bastida Adrián ${ }^{1^{*}}$}

${ }^{1}$ Universidad Autónoma del Estado de Hidalgo. Instituto de Ciencias Agropecuarias. México. ${ }^{2}$ Universidad
del Papaloapan, Campus Tuxtepec. México. ${ }^{3}$ Universidad Autónoma del Estado de México. Facultad de
Medicina Veterinaria y Zootecnia. El Cerrillo Piedras Blancas. Toluca, Estado de México. C.P 50090.
${ }^{4}$ Unidad Académica de Ciencias Biológicas. Universidad Autónoma de Zacatecas. Zacatecas, México.
${ }^{5}$ Facultad de Ciencias Veterinarias, Universidad Austral de Chile, Isla Teja s/n, Casilla 567, Valdivia, Chile
${ }^{*}$ Autor responsable y de correspondencia: Nallely Rivero-Perez, Zaragoza-Bastida Adrian. Rancho
Universitario Av. Universidad km 1, A.P. 32 CP.43600. 01771717 2000 ext. 2440. Ex-Hda. de Aquetzalpa,
Hidalgo, México. ubaldolizet8@gmail.com, j_lizbeth_1895@hotmail.com, benvac2004@yahoo.com.mx,
vvo@uaemex.mx, delgadillolucia@gmail.com, crosenfe@uach.cl, nallely_rivero@uaeh.edu.mx, adrian_zaragoza@uaeh.edu.mx.

\section{RESUMEN}

La Organización Mundial de Sanidad Animal (OIE) menciona que el uso inadecuado de antibióticos ha propiciado la aparición de cepas bacterias resistentes a los mecanismos de acción de estos fármacos, debido a la escasez de terapias efectivas se requiere del desarrollo de nuevas opciones de tratamiento para enfermedades que afectan la salud, al respecto los extractos de plantas o bien sus compuestos puros ofrecen una alternativa. El objetivo de la presente investigación fue caracterizar y evaluar la actividad antibacteriana in vitro del extracto hidroalcohólico de Croton draco sobre bacterias de importancia sanitaria. El extracto se obtuvo mediante maceración hidroalcohólica, se realizó una caracterización cualitativa y química del extracto, la actividad antibacteriana se determinó mediante la Concentración Mínima Inhibitoria (CMI) y la Concentración Mínima Bactericida (CMB). Los ensayos de caracterización cualitativa realizados al extracto hidroalcohólico de Croton draco indican la presencia de compuestos fenólicos, terpenos, saponinas y alcaloides, por cromatografía de gases se determinó por primera vez la presencia de timol y carvacrol en concentraciones de $0.5340 \mathrm{mg} / \mathrm{ml}$ y $0.4206 \mathrm{mg} / \mathrm{mL}$ respectivamente. Las bacterias mostraron diferentes grados de sensibilidad, sin embargo, se determinó una mayor actividad frente a bacterias Gram positivas como $L$. monocytogenes, $S$. aureus y $B$. subtillis.

Palabras clave: Croton draco, Extracto hidroalcohólico, Antibacteriano, Concentración Mínima Inhibitoria, Concentración Mínima Bactericida.

\begin{abstract}
The World Organization for Animal Health (OIE) mentions that the inappropriate use of antibiotics has led to the emergence of strains of bacteria resistant to the mechanisms of action of these drugs, due to the scarcity of effective therapies, the development of new treatment options is required for diseases that affect health, in this respect plant extracts or their pure compounds offer an alternative. The aim of the present investigation was to characterize and evaluate the in vitro antibacterial activity of the hydroalcoholic extract of Croton draco on bacteria of sanitary importance. The extract was obtained by hydroalcoholic maceration, a qualitative and chemical characterization of the extract was carried out, and the antibacterial activity was determined by the Minimum Inhibitory Concentration (MIC) and the Minimum Bactericidal Concentration
\end{abstract}


(MBC). The qualitative characterization tests performed on the hydroalcoholic extract of Croton draco indicate the presence of phenolic compounds, terpenes, saponins and alkaloids, by gas chromatography the presence of thymol and carvacrol was determined in concentrations of $0.5340 \mathrm{mg} / \mathrm{ml}$ and $0.4206 \mathrm{mg} / \mathrm{mL}$ respectively. The bacteria showed different degrees of sensitivity to the hydroalcoholic extract, however, greater activity was determined against Gram positive bacteria such as Listeria monocytogenes, Staphylococcus aureus and Bacillus subtilis.

Keywords: Croton draco, Hydroalcoholic extract, Antibacterial, Minimum Inhibitory Concentration, Minimum Bactericidal Concentration.

\section{INTRODUCCIÓN}

La Organización Mundial de Sanidad Animal (OIE) menciona que el uso inadecuado de antibióticos, ha propiciado la aparición de cepas bacterias resistentes a los mecanismos de acción de estos fármacos, esto supone una situación alarmante tanto para la salud humana como para la sanidad animal (OIE, 2016), debido a la escasez de terapias efectivas. Se requiere del desarrollo de nuevas opciones de tratamiento para enfermedades que afectan la salud, los extractos de plantas o bien sus compuestos puros ofrecen una alternativa potencial para el desarrollo de nuevos tratamientos antimicrobianos que puedan ser utilizadas para el control de microorganismos patógenos, debido a la presencia de metabolitos o compuestos que inhiben el crecimiento o provocan la muerte de estos (Lavor et al., 2014; Upadhyay et al., 2016).

Al respecto Maxia et al., (2005) mencionan que existen prácticas etnoveterinarias por parte de ganaderos y pastores, basadas en conocimientos transmitidos de generación en generación. Por su parte Martínez and Jiménez-Escobar (2017), documentaron un total de 62 prácticas etnoveterinarias; destacando el uso de las plantas para el tratamiento de heridas, afecciones oculares y digestivas, prácticas asociadas a 44 especies vegetales pertenecientes a 30 familias botánicas; entre las que destaca el género Croton (Croton lanatus).

La sangre de dragón, sangregado o sangre de drago (Croton draco), es uno de los arbustos utilizados en la medicina tradicional por diferentes culturas del mundo; algunos de sus usos etnomedicinales, son: hemostático, antidiarreico, antiulceroso, antiviral, cicatrizante, antitumoral, antiinflamatorio, antioxidante y antimicrobiano, entre otros (Gupta et al., 2008).

En México a esta planta se le atribuyen una gran cantidad de propiedades curativas, como las mencionadas anteriormente, debido a la amplia gama de metabolitos secundarios que posee, como: alcaloides, taninos, diterpenos y aceites volátiles (Salatino et al., 2007); sin embargo, se requiere de estudios para determinar las concentraciones a las cuales presenta su efecto farmacológico y los compuestos a los cuales se les puedan atribuir las propiedades farmacológicas.

Estudios realizados por Peres et al., (1997), demuestran que los extractos de algunas especies de Croton presentan actividad inhibitoria frente a Staphylococcus aureus y Salmonella typhimuryum. 
De acuerdo a lo mencionado anteriormente, el objetivo de la presente investigación fue realizar una caracterización cualitativa y química del extracto hidroalcohólico de Crotón draco, así como determinar su actividad antibacteriana (in vitro) sobre bacterias de importancia sanitaria.

\section{Obtención del extracto}

\section{MATERIAL Y MÉTODOS}

Se recolectaron muestras de material vegetal de la parte aérea de Crotón draco durante el verano (junio-agosto) en el municipio de Huatusco $\left(19^{\circ} 08^{\prime} 56^{\prime \prime} \mathrm{N}\right.$ 96 $\left.6^{\circ} 57^{\prime} 58^{\prime \prime} \mathrm{O}\right)$ perteneciente al estado de Veracruz; para la identificación de la planta se consultó el herbario de la Universidad Nacional Autónoma de México, y el espécimen vegetal se identificó como Crotón draco subsp. draco (IBUNAM:MEXU:501697).

El material vegetal fue secado a temperatura ambiente en ausencia de luz, se maceraron $250 \mathrm{~g}$ del material seco (triturado) en un litro de solución hidroalcohólica (70:30, agua: metanol), durante 48 horas a temperatura ambiente en ausencia de luz. Se filtró el extracto líquido de la maceración mediante papel filtro y algodón; posteriormente el líquido obtenido fue concentrado a presión reducida en un rotavapor (BÜCHI ${ }^{\mathrm{T} M} \mathrm{R}-210$, Flawil, Germany), de acuerdo a la metodología descrita por (Rivero-Pérez et al., 2016). El extracto resultante fue conservado en refrigeración hasta su posterior evaluación.

\section{Caracterización química del extracto}

Al extracto hidroalcohólico de Crotón draco, se le realizó el perfil químico cualitativo de acuerdo al procedimiento descrito por Bañuelos-Valenzuela et al., (2018), en tubos de ensayo Pyrex de $10 \mathrm{ml}$. Las pruebas fueron las siguientes: prueba del $\mathrm{KMnO}_{4}$ para insaturaciones, prueba del $\mathrm{FeCl} 3$ para oxidrilos fenólicos (taninos vegetales), prueba de Liebermann-Burchard para esteroles y triterpenos, prueba de Salkowski para esteroles y triterpenos, prueba de cumarinas, prueba de Baljet para sesquiterpenlactonas, prueba del $\mathrm{H}_{2} \mathrm{SO}_{4}$ para flavonoides, prueba de Shinoda para flavonoides, prueba de Dragendorff para alcaloides, prueba de taninos, prueba de floratanino, prueba de esteroides, prueba de la agitación, prueba del bicarbonato de sodio y prueba de Salkowski para saponinas.

La composición química del extracto hidroalcohólico de Crotón draco se determinó de acuerdo a la metodología descrita por Bañuelos-Valenzuela et al., (2018), mediante un cromatógrafo de gases (CG; Agilent Tecnologies serie $6890 \mathrm{~N}$ fabricado en U.S.A), con una columna polar DB_WAXetr, a $250{ }^{\circ} \mathrm{C}$ y $12.13 \mathrm{psi}$, con un flujo de He $36.5 \mathrm{ml}$ min-1 después de la inyección. Las condiciones para la columna fueron: temperatura inicial 50 ${ }^{\circ} \mathrm{C}$ de cero a dos min, aumentando de 10 en $10^{\circ} \mathrm{C}$ hasta llegar a $250{ }^{\circ} \mathrm{C}$, manteniendo la temperatura constante por 5 min para luego descender a $50{ }^{\circ} \mathrm{C}$ por dos min con un flujo de He de $1.6 \mathrm{ml}$ min-1 a una presión de 12.13 psi y una velocidad promedio de $25 \mathrm{~cm} \mathrm{~s}$ 1 , utilizando un detector de flama ionizante (FID), a una temperatura de $210^{\circ} \mathrm{C}$, con un 
flujo de $\mathrm{H} 2$ de $40 \mathrm{ml}$ min-1 y un flujo de aire de $450 \mathrm{ml}$ min-1. Los estándares (SigmaAldrich) se utilizaron en concentraciones diferentes (cuadro 1).

\begin{tabular}{|c|c|c|c|c|c|}
\hline Estándar & $\begin{array}{l}\text { Timol } \\
\left(\mathrm{mg} \mathrm{ml}^{-1}\right)\end{array}$ & $\begin{array}{l}\text { Carvacrol } \\
\left(\mathrm{mg} \mathrm{ml}^{-1}\right)\end{array}$ & $\begin{array}{l}\text { Linalol } \\
\left(\mathrm{mg} \mathrm{ml}^{-1}\right)\end{array}$ & $\begin{array}{l}\text { Terpineno } \\
\left(\mathrm{mg} \mathrm{ml}^{-1}\right)\end{array}$ & $\begin{array}{l}\text { Limoneno } \\
\left(\mathrm{mg} \mathrm{ml}^{-1}\right)\end{array}$ \\
\hline 1 & 10.373 & 8.284 & 7.744 & 7.154 & 8.496 \\
\hline 2 & 5.186 & 4.142 & 3.872 & 3.577 & 4.248 \\
\hline 3 & 2.593 & 2.071 & 1.936 & 1.789 & 2.124 \\
\hline 4 & 1.297 & 1.035 & 0.968 & 0.894 & 1.062 \\
\hline 5 & 0.648 & 0.518 & 0.484 & 0.447 & 0.531 \\
\hline 6 & 0.324 & 0.259 & 0.242 & 0.224 & 0.265 \\
\hline
\end{tabular}

\section{Actividad antimicrobiana}

La actividad antibacteriana se determinó mediante la Concentración Mínima Inhibitoria (CMI) y la Concentración Mínima Bactericida (CMB) para el extracto, siguiendo las especificaciones del CLSI., (2012). Las pruebas de actividad antimicrobiana se llevaron a cabo con las cepas ATCC, Staphylococcus aureus ATCC ${ }^{6538}$, Escherichia coli ATCC 35218 , Pseudomona aeruginosa ATCC 9027, Salmonella typhi ATCC ${ }^{14028}$, Salmonella cholerasuis ATCC ${ }^{10708}$, Listeria monocytogenes ATCC ${ }^{19113}$ y Bacillus subtilis ATCC ${ }^{6633}$, las cuales fueron reactivadas en Agar Muller Hinton (BD Bioxon), se corroboró la pureza de las cepas por medio de la tinción de Gram.

Una vez confirmada la morfología de la bacteria se inoculó una colonia en caldo nutritivo (BD Bioxon), el cual fue incubado en agitación constante (70rpm) por 24 horas a $37^{\circ} \mathrm{C}$. Trascurrido el tiempo de incubación, el inóculo se ajustó con caldo nutritivo al 0.5 del patrón de turbidez de McFarland (Remel, R20421), el cual corresponde a $150 \times 10^{6} \mathrm{cel} / \mathrm{ml}$. Para la determinación de la Concentración Mínima Inhibitoria (CMI) se utilizó el método de microdilución en placa; las concentraciones evaluadas fueron: 400, 200 100, 50, 25, $12.50,6.25,3.12 \mathrm{mg} / \mathrm{ml}$, para las cepas a evaluar. Cada concentración fue preparada con caldo nutritivo (BD Bioxon). El tratamiento se realizó por triplicado en una placa de 96 pozos, se colocaron $100 \mu \mathrm{l}$ de cada una de las diluciones más $10 \mu \mathrm{l}$ de la suspensión bacteriana previamente ajustada a 0.5 de McFarland. Una vez realizada la inoculación la placa se incubó a $37^{\circ} \mathrm{C}$ durante 24 horas a $70 \mathrm{rpm}$ en agitación constante. El control positivo fue Kanamicina (AppliChem 4K10421) a concentraciones de 64, 32, 16, 8.0, 4.0, 2.0, 1.0 y $0.5 \mu \mathrm{g} / \mathrm{ml}$. Se utilizó caldo nutritivo como control negativo.

Para determinar el punto final de la $\mathrm{CMI}$ se empleó un método colorimétrico basado en el uso de sales de tetrazolium, según lo descrito por Balouiri et al., (2016). Una vez transcurrido el tiempo de incubación se agregaron $20 \mu \mathrm{l}$ de una solución al $0.04 \%(\mathrm{w} / \mathrm{v})$ de p-iodonitrotetrazolium (Sigma-Aldrich 18377) en cada pozo; se incubó por 30 minutos a $37^{\circ} \mathrm{C}$ y se realizó la lectura, determinándose como la concentración mínima inhibitoria; aquella a la cual la solución vira a rosa (Kaewpiboon et al., 2012; Mothana et al., 2009). Para determinar la Concentración Mínima Bactericida (CMB), previa adición del piodonitrotetrazolium, se inocularon $5 \mu \mathrm{l}$ de cada pozo en agar Mueller Hinton, para 
posteriormente incubar a $37^{\circ} \mathrm{C}$ durante 24 horas. Trascurrido el tiempo de incubación se verificó el crecimiento de las bacterias para determinar la concentración mínima bactericida del extracto, fracción o metabolito; considerándose como $\mathrm{CMB}$, la concentración a la cual no se observó crecimiento bacteriano en la placa.

\section{Análisis estadístico}

Los datos obtenidos fueron normalizados y analizados mediante un análisis de varianza y una comparación de medias por Tukey, a un nivel de confianza del $95 \%$; con el paquete estadístico Minitab 18.

\section{RESULTADOS}

\section{Caracterización química del extracto hidroalcohólico de Crotón draco}

Los ensayos de caracterización cualitativa realizados al extracto hidroalcohólico de Crotón draco, indican la presencia de compuestos fenólicos (cumarinas, flavonoides y flavonas), terpenos (sesquiterpenos), saponinas y alcaloides.

El análisis en el cromatógrafo de gases del extracto hidroalcohólico de Crotón draco determinó la presencia de timol y carvacrol en concentraciones de $0.5340 \mathrm{mg} / \mathrm{ml}$ y 0.4206 $\mathrm{mg} / \mathrm{ml}$ respectivamente; sin determinar la presencia de Linalol, terpineno y limoneno.

\section{Actividad antibacteriana}

En la Concentración Mínima Inhibitoria (CMI) del extracto de hidroalcohólico de Crotón draco, se determinaron diferencias estadísticas significativas $(P \leq 0.05)$ entre las bacterias evaluadas, la CMI para E. coli, S. typhi, S. cholerasuis, $P$. aeruginosa fue de $100 \mathrm{mg} / \mathrm{ml}$; sin encontrar diferencias estadísticas, para L. monocytogenes de $50 \mathrm{mg} / \mathrm{ml}$ con diferencias significativas con respecto al resto de las bacterias evaluadas y de $25 \mathrm{mg} / \mathrm{ml}$ para $S$. aureus, B. subtillis; mostrando diferencias estadísticas con las bacterias antes mencionadas. Por otra parte, las CMI para las bacterias evaluadas mostraron diferencias estadísticas significativas con respecto a la CMI del control con Kanamicina, como se muestra en el cuadro 2.

Cuadro 2. Concentración Mínima Inhibitoria y Concentración Mínima Bactericida del extracto hidroalcohólico de Crotón draco, sobre bacterias de importancia sanitaria

\begin{tabular}{lcccc}
\hline & \multicolumn{2}{c}{ Concentración Mínima Inhibitoria } & \multicolumn{2}{c}{ Concentración Mínima Bactericida } \\
\hline Bacteria evaluada & EHCD $(\mathbf{m g} / \mathbf{m L})$ & Kanamicina $(\mu \mathbf{g} / \mathbf{m L})$ & EHCD $(\mathbf{m g} / \mathbf{m L})$ & Kanamicina $(\mu \mathrm{g} / \mathbf{m L})$ \\
\hline E. coli & $100^{\mathrm{c}}$ & 4 & $200^{\mathrm{c}}$ & 8 \\
S. typhi & $100^{\mathrm{c}}$ & 4 & $200^{\mathrm{c}}$ & 8 \\
S.cholerasuis & $100^{\mathrm{c}}$ & 2 & $200^{\mathrm{c}}$ & 4 \\
P. aeruginosa & $100^{\mathrm{c}}$ & 16 & $200^{\mathrm{c}}$ & 32 \\
L. monocytogenes & $50^{\mathrm{b}}$ & 2 & $100^{\mathrm{b}}$ & 4 \\
B. subtillis & $25^{\mathrm{a}}$ & 0.5 & $50^{\mathrm{a}}$ & 1 \\
S. aureus & $25^{\mathrm{a}}$ & 2 & $50^{\mathrm{a}}$ & 4 \\
$P$ value & 0.0001 & & 0.0001 & \\
\hline
\end{tabular}

EHCD; extracto hidroalcóholico de Croton draco, diferentes literales ${ }^{\mathrm{a}, \mathrm{b}, \mathrm{c}}$ en las columnas indican diferencias estadísticas significativas $(P \leq 0.05)$ 
En la Concentración Mínima Bactericida (CMB) del extracto de hidroalcohólico de Crotón draco, se determinaron diferencias estadísticas significativas $(p \geq 0.05)$ entre las bacterias evaluadas, la CMB para E. coli, S. typhi, S. cholerasuis, $P$. aeruginosa fue de $200 \mathrm{mg} / \mathrm{ml}$; sin encontrar diferencias estadísticas entre estas, para $L$. monocytogenes de $100 \mathrm{mg} / \mathrm{ml}$ con diferencias significativas con respecto al resto de las bacterias evaluadas, y de 50 $\mathrm{mg} / \mathrm{ml}$ para $S$. aureus, B. subtillis; mostrando diferencias estadísticas con las bacterias antes mencionadas. Por otra parte, las CMB para las bacterias evaluadas mostraron diferencias estadísticas significativas $(P \leq 0.05)$ con respecto a la $\mathrm{CMB}$ del control positivo (Kanamicina), como se muestra en el cuadro 2.

\section{DISCUSIÓN}

Actualmente, la aparición de bacterias resistentes o multirrestentes a fármaco antibacterianos, representa un grave problema de salud pública a nivel mundial; esta problemática requiere una vigilancia constante, situación que desafía a la comunidad científica para la búsqueda de alternativas (Lai et al., 2017). La disminución de la eficacia y el aumento de la toxicidad de los antimicrobianos sintéticos agravan el problema; por lo que los científicos están buscando compuestos naturales para obtener soluciones. Al respecto la medicina tradicional basada en el uso de plantas medicinales representan una alternativa para el tratamiento de enfermedades causadas por bacterias resistentes o multirrestentes, tanto en humanos como animales (Valle et al., 2015).

Los resultados de la caracterización cualitativa del extracto hidroalcohólico de Crotón draco, indica la presencia de compuestos fenólicos (cumarinas, flavonoides y flavonas), terpenos (sesquiterpenos), saponinas y alcaloides; metabolitos que han sido reportados en otras plantas con actividad antibacteriana, según la literatura: cumarinas (Souzaa et al., 2005), flavonoides (Cushnie et al., 2005), sesquiterpenos (Barrero et al., 2005), saponinas (Mandal et al., 2005) y alcaloides (Cushnie et al., 2014).

Por otro lado, la caracterización química por medio de cromatografía de gases, permitió determinar la presencia de timol $(0.5340 \mathrm{mg} / \mathrm{ml})$ y carvacrol $(0.4206 \mathrm{mg} / \mathrm{ml})$; compuestos con actividad antibacteriana de acuerdo con Du et al., (2005).

Salatino et al., (2007) reportó una caracterización cualitativa y química de diferentes especies del género Crotón, la cual coincide con la caracterización del extracto evaluado en el presente experimento.

Peres et al., (1997) reportó la actividad antibacteriana del extracto metanólico de Crotón urucurana; determinado una mejor actividad del extracto contra bacterias Gram positivas (Staphylococcus aureus). Resultado que concuerdan con los obtenidos en la presente investigación, ya que las concentraciones más bajas de CMI y CMB fueron frente a las cepas Gram positivas de L. monocytogenes (50 mg/ml CMl, $100 \mathrm{mg} / \mathrm{ml} \mathrm{CMB),} \mathrm{B.} \mathrm{subtillis}$ y $S$. aureus $(25 \mathrm{mg} / \mathrm{ml} \mathrm{CMl,} 50 \mathrm{mg} / \mathrm{ml} \mathrm{CMB})$. Lo cual puede explicarse debido a que la 
membrana externa de las bacterias Gram negativas actúa como una barrera selectiva que limita la entrada de antibacterianos (Cabrera et al., 2007).

Estudios similares realizados por Selowa et al., (2009), reportan la actividad antibacteriana de los extractos metanolicos de Crotón salvatycus, $C$. megalobotrys, $C$. steenkapianus, contra E. coli, S. aureus y P. aeruginosa, siendo C. steenkapianus; el único inactivo frente a $E$. coli y $S$. aureus. Caso contrario a Crotón draco, el cual mantiene actividad frente a estas especies bacterianas; esta diferencia puede deberse a que los compuestos y las concentraciones de los mismos cambian dependiendo del género, especie, estado fenológico, situaciones de estrés y localización geográfica de la planta; además de la técnica utilizada para la extracción y concentración de metabolitos secundarios (Hernández-Alvarado et al., 2018).

Aunque en el presente experimento no se determinó el mecanismo de acción, el mecanismo de acción al cual se asocia la actividad antibacteriana de las cumarinas, flavonoides, sesquiterpenos, saponinas y alcaloides, se debe a su capacidad para afectar la permeabilidad de la membrana celular de las bacterias Gram positivas y Gram negativas; disminuir el pH citoplasmático y causar una hiperpolarización de la membrana celular, de acuerdo con lo publicado por Gonelimali et al., 2018.

\section{CONCLUSIÓN}

Los resultados indican que en el extracto hidroalcohólico de Crotón draco, contiene metabolitos con potencial actividad antibacteriana; abriendo así la posibilidad de ser utilizado como un fitofármaco capaz de actuar frente a bacterias de importancia sanitaria; sin embargo, se recomienda en estudios futuros aislar y evaluar el o los compuestos que le confieren dicha actividad con cepas de colección y cepas aisladas de campo con resistencia a diferentes antibióticos.

\section{LITERATURA CITADA}

BALOUIRI M, Sadiki M, Ibnsouda SK. 2016. Methods for in vitro evaluating antimicrobial activity: A review. Journal of Pharmaceutical Analysis. 6(2):71-79. https://doi.org/10.1016/j.jpha.2015.11.005

BAÑUELOS-VALENZUELA R, Delgadillo L, Chairez F, Delgadillo O, Meza-López C. 2018. Composición química y FTIR de extractos etanólicos de Larrea tridentata, Origanum vulgare, Artemisa ludoviciana y Ruta graveolens. Agrociencia. 52(3): 309-321. https://dialnet.unirioja.es/servlet/articulo?codigo $=6423180$. 
BARRERO AF, Quílez del Moral JF, Lara A, Herrador MM. 2005. Antimicrobial Activity of Sesquiterpenes from the Essential Oil of Juniperus thurifera Wood. Planta Med. 71(01): 67-71. https://doi:10.1055/s-2005-837753

CABRERA CE, Gómez RF, Zúñiga AE. 2007. La resistencia de bacterias a antibióticos, antisépticos y desinfectantes una manifestación de los mecanismos de supervivencia y adaptación. Colombia Médica. 38 (2): 149-158.

http://www.scielo.org.co/pdf/cm/v38n2/v38n2a07.pdf

CLSI (Clinical and Laboratory Standards Institute). 2012. Methods for Dilution Antimicrobial Susceptibility Tests for Bacteria That Grow Aerobically; Approved StandardNinth Edition. USA. Pp. 88.

CUSHNIE TPT, Lamb AJ. 2005. Antimicrobial activity of flavonoids. International Journal of Antimicrobial Agents. 26(5): 343-356. https://doi.org/10.1016/j.ijantimicag.2005.09.002

CUSHNIE TPT, Cushnie B, Lamb AJ. 2014. Alkaloids: An overview of their antibacterial, antibiotic-enhancing and antivirulence activities. International Journal of Antimicrobial Agents. 44(5):377-386. https://doi:https://doi.org/10.1016/j.ijantimicag.2014.06.001

DU E, Gan L, Li Z, Wang W, Liu D, Guo Y. 2015. In vitro antibacterial activity of thymol and carvacrol and their effects on broiler chickens challenged with Clostridium perfringens. Journal of animal science and biotechnology. 6:58-58. https://doi:10.1186/s40104-015-0055-7.

GONELIMALI FD, Lin J, Miao W, Xuan J, Charles F, Chen M, Hatab SR. 2018. Antimicrobial Properties and Mechanism of Action of Some Plant Extracts Against Food Pathogens and Spoilage Microorganisms. Frontiers in microbiology. 9: 1639-1639. https://doi:10.3389/fmicb.2018.01639

GUPTA D, Bleakley B, Gupta, RK. 2008. Dragon's blood: Botany, chemistry and therapeutic uses. Journal of Ethnopharmacology. 115(3):361-380.

doi: https://doi.org/10.1016/j.jep.2007.10.018

HERNÁNDEZ-ALVARADO JL, Zaragoza-Bastida A, López-Rodríguez GM, Peláez-Acero A, Olmedo-Juárez A, Rivero-Perez N. 2018. Actividad antibacteriana y sobre nematodos gastrointestinales de metabolitos secundarios vegetales: enfoque en Medicina Veterinaria. Abanico veterinario. 8(1): 14-27. https://dx.doi.org/10.21929/abavet2018.81.1

KAEWPIBOON C, Lirdprapamongkol K, Srisomsap C, Winayanuwattikun P, Yongvanich, T, Puwaprisirisan, Puwaprisirisan P,Svasti J, Assavalapsakul W. 2012. Studies of the in vitro cytotoxic, antioxidant, lipase inhibitory and antimicrobial activities of selected Thai 
medicinal plants. BMC Complementary and Alternative Medicine. 12(1): 217. https://doi.org/10.1186/1472-6882-12-217.

LAI CC, Lee K, Xiao Y, Ahmad N, Veeraraghavan B, Thamlikitkul V, Hsueh PR. 2014. High burden of antimicrobial drug resistance in Asia. Journal of Global Antimicrobial Resistance. 2(3): 141-147. https://doi.org/10.1016/j.jgar.2014.02.007.

LAVOR AK LS, Matias EFF, Alves EF, Santos BS, Figueredo FG, Lima LF, Coutinho HDM. 2014. Association between drugs and herbal products: In vitro enhancement of the antibiotic activity by fractions from leaves of Croton campestris A. (Euphorbiaceae). European Journal of Integrative Medicine. 6(3):301-306.

https://doi.org/10.1016/j.eujim.2014.03.002.

MANDAL P, Sinha BSP, Mandal NC. 2005. Antimicrobial activity of saponins from Acacia auriculiformis. Fitoterapia. 76(5): 462-465. https://doi.org/10.1016/j.fitote.2005.03.004.

MARTÍNEZ GJ, Jiménez - Escobar ND. 2017. Plantas de interés veterinario en la cultura campesina de la Sierra de Ancasti (Catamarca, Argentina). Boletín Latinoamericano y del Caribe de Plantas Medicinales y Aromáticas. 16(4):329-346. https://www.blacpma.usach.cl/sites/blacpma/files/articulo_1_-_1292_-_329_-_346_0.pdf

MAXIA A, Lancioni C, Mura L, Mongiano PD. 2005. Plantas usadas en la practica etnoveterinaria en el Nuorese (Cerdena, Italia). Revista de Fitoterapia. 5(2). 155-161. https://docplayer.es/6533436-Plantas-usadas-en-la-practica-etnoveterinaria-en-elnuorese-cerdena-italia.html

MOTHANA RA, Lindequist U, Gruenert R, Bednarski PJ. 2009. Studies of the in vitro anticancer, antimicrobial and antioxidant potentials of selected Yemeni medicinal plants from the island Soqotra. BMC complementary and alternative medicine. 9(1):7. https://doi.org/10.1186/1472-6882-9-7.

OIE (Organización Internacional de Salud Animal). 2016. Estrategia de la OIE sobre la resistencia a los agentes antimicrobianos y su uso prudente. www.oie.int/fileadmin/Home/esp/Media_Center/docs/pdf/PortalAMR/ES_OIE-

AMRstrategy.pdf

PERES, MTLP, Monache FD, Cruz AB, Pizzolatti MG, Yunes RA. 1997. Chemical composition and antimicrobial activity of Croton urucurana Baillon (Euphorbiaceae). Journal of Ethnopharmacology. 56(3):223-226. https://doi.org/10.1016/S03788741(97)00039-1

RIVERO-PEREZ N, Ayala-Martínez M, Zepeda-Bastida A, Meneses-Mayo M, OjedaRamírez D. 2016. Anti-inflammatory effect of aqueous extracts of spent Pleurotus 
ostreatus substrates in mouse ears treated with 12-O-tetradecanoylphorbol-13-acetate. Indian Journal of Pharmacology. 48(2):141-144. https://doi.org/10.4103/02537613.178826.

SALATINO A, Salatino MLF, Negri G. 2007. Traditional uses, chemistry and pharmacology of Croton species (Euphorbiaceae). Journal of the Brazilian Chemical Society. 18:11-33. http://dx.doi.org/10.1590/S0103-50532007000100002

SELOWA SC, Shai LJ, Masoko P, Mokgotho MP, Magano SR. 2009. Antibacterial activity of extracts of three Croton species collected in Mpumalanga region in South Africa. African Journal of Traditional Complementary and Alternative Medicine. 7(2):98-103. https://www.ncbi.nlm.nih.gov/pmc/articles/PMC3021162/pdf/AJTCAM0702-0098.pdf

SOUZAA SMD, Monacheb FD, Smania A. 2005. Antibacterial Activity of Coumarins. Naturforsch. 60: 693-700.

https://pdfs.semanticscholar.org/d5a6/502543e2174bd3da1f1d6bb6d73b98784b4e.pdf

UPADHYAY A, Karumathil DP, Upadhyaya I, Bhattaram V, Venkitanarayanan K. 2016. Controlling Bacterial Antibiotic Resistance Using Plant-Derived Antimicrobials. En: Kateryna K, Mahendra R. Antibiotic Resistance. Academic Press. Pp. 205-226. ISBN 9780128036426. https://doi.org/10.1016/B978-0-12-803642-6.00010-1.

VALLE DL, Andrade JI, Puzon JJM, Cabrera EC, Rivera WL. 2015. Antibacterial activities of ethanol extracts of Philippine medicinal plants against multidrug-resistant bacteria. Asian Pacific Journal of Tropical Biomedicine. 5(7): 532-540. https://doi.org/10.1016/j.apjtb.2015.04.005 